\title{
Arrhythmogenic Cardiomyopathy of the Right Ventricle. Predictive Value of QT Interval Dispersion to Assess Arrhythmogenic Risk and Sudden Death
}

\author{
Márcio L. A. Fagundes, Ivan G. Maia, Fernando E. S. Cruz Fo, Paulo A. G. Alves, Silvia H.Boghossian, \\ José Carlos Ribeiro, Roberto Sá \\ Rio de Janeiro, RJ - Brazil
}

\begin{abstract}
Objective - To determine in arrhythmogenic right ventricular cardiomyopathy the value of QT interval dispersion for identifying the induction of sustained ventricular tachycardia in the electrophysiological study or the risk of sudden cardiac death.
\end{abstract}

Methods - We assessed QT interval dispersion in the 12lead electrocardiogram of 26 patients with arrhythmogenic right ventricular cardiomyopathy. We analyzed its association with sustained ventricular tachycardia and sudden cardiac death, and in 16 controls similar in age and sex.

Results - (mean $\pm S D)$. QT interval dispersion: patients $=53.8 \pm 14.1 \mathrm{~ms} ;$ control group $=35.0 \pm 10.6 \mathrm{~ms}, p=0.001$. Patients with induction of ventricular tachycardia: $52.5 \pm 13.8 \mathrm{~ms}$; without induction of ventricular tachycardia: $57.5 \pm 12.8 \mathrm{~ms}, p=0.420$. In a mean follow-up period of $41 \pm 11$ months, five sudden cardiac deaths occurred. QT interval

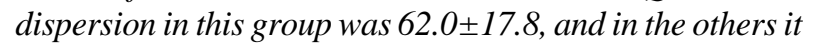
was $51.9 \pm 12.8 \mathrm{~ms}, p=0.852$. Using a cutoff $\geq 60 \mathrm{~ms}$ to define an increase in the degree of the QT interval dispersion, we were able to identify patients at risk of sudden cardiac death with a sensitivity of $60 \%$, a specificity of $57 \%$, and positive and negative predictive values of $25 \%$ and $85 \%$, respectively.

Conclusion - Patients with arrhythmogenic right ventricular cardiomyopathy have a significant increase in the degree of $Q T$ interval dispersion when compared with the healthy population. However it, did not identify patients with induction of ventricular tachycardia in the electrophysiological study, showing a very low predictive value for defining the risk of sudden cardiac death in the population studied.

Keywords: sudden death, arrhythmogenic dysplasia/ cardiomyopathy, QT interval dispersion

Hospital PróCardíaco - Rio de Janeiro

Mailing address: Márcio L. A. Fagundes - Hospital PróCardíaco - Rua Dona Mariana, 219 - 22280-020 - Rio de Janeiro, RJ, Brazil
Arrhythmogenic right ventricular cardiomyopathy is a familial disease in which a progressive replacement of the healthy myocardial fibers by fatty tissue takes place ${ }^{1,2}$. It causes important myocardial structure disarrangement with apparently healthy tissue intermingled with fatty that tissue determines electrical conditions favoring an arrhythmogenic substrate. Sustained and nonsustained arrhythmias observed in this disease have a characteristic morphology of the left bundle-branch block type because the arrhythmogenic focus is located in the right ventricle ${ }^{3}$.

With the advances in diagnostic tools and a better knowledge of the clinical profile of arrhythmogenic right ventricular cardiomyopathy, an increasing number of cases of this condition have been diagnosed. Arrhythmogenic right ventricular cardiomyopathy, however, is still a significant finding in autopsies of patients under the age of 50 years who die of sudden death, many of whom were without a previous diagnosis. This incidence has ranged from $5 \%$ to $20 \%$, depending on the regional prevalence of the disease $^{4,5}$.

Studies have showed a marked variation in the incidence of sudden death in arrhythmogenic right ventricular cardiomyopathy. Its presence, however, is especially related to physical exercise, occurring even in asymptomatic patients or in those whose symptoms are only related to ventricular tachyarrhythmias. The clinical history of syncopal episodes or recovery from cardiopulmonary arrest are known factors of poor prognosis. Variables obtained through invasive and noninvasive methods have not been very useful in defining the population at a greater risk $^{6,7}$.

Recently, a new noninvasive method for stratifying the risk of arrhythmogenic sudden death was introduced based on the determination of the degree of dispersion of ventricular repolarization observed among the values of the QT interval of an electrocardiogram (QTDp) ${ }^{8}$. These results were assessed in ischemic heart disease ${ }^{9,10}$, but no study, 
however, exists that attempts to define the applicability of the method in arrhythmogenic right ventricular cardiomyopathy, which was the objective of the present study.

\section{Methods}

We selected 26 patients out of a total of 67 patients being clinically followed up with arrhythmias very probably originating in the right ventricle (morphology of the left bundle-branch block type without septal or left ventricular alterations on the echocardiogram). These patients had the following characteristics: 1 ) presence of sinus rhythm and well defined $T$ waves in at least 10 of the 12 electrocardiographic leads; 2) presence of arrhythmogenic potentials at the end of the QRS complexes on the signal-averaged electrocardiogram ${ }^{11,12} ; 3$ ) presence of structural changes in the right ventricle on the echocardiogram, such as global or segmentary (outflow tract) cavitary dilation, microaneurys$\mathrm{ms}$ or sacculations in the outflow tract or ventricular inlet, hyperrefringence of the moderate band ${ }^{13}$;4) magnetic resonance imaging depicting thinning of the cavitary wall or fatty infiltration, or both ${ }^{14}$.

Even though 3 more patients had arrhythmogenic right ventricular cardiomyopathy, they were excluded from the study because they did not meet criterion 1 .

Out of the 26 patients, 20 were males, and the mean age was $34 \pm 10.9$ years. All patients were in NYHA functional class I or II and had in the 24-hour Holter nonsustained ventricular tachyarrhythmias with a number of isolated extrasystoles higher than 10 per hour (>240 in 24 hours). During clinical follow-up, 19 patients experienced at least one episode of sustained ventricular tachycardia. All these sustained and nonsustained arrhythmias had the classical morphology of the left bundle-branch block type. Table I shows the clinical data of the patients.

The initial electrocardiogram raising the diagnostic suspicion was selected for analyzing the QT interval dispersion. Therefore, no patient was on medication. All recor-

\begin{tabular}{|c|c|c|c|c|c|c|c|}
\hline \multicolumn{8}{|c|}{ Table I - Individualized data of the patients } \\
\hline Case & Sex & Age & $\begin{array}{c}\text { Ventricular } \\
\text { Arrhythmia }\end{array}$ & Case & Sex & Age & $\begin{array}{l}\text { Ventricular } \\
\text { Arrhythmia }\end{array}$ \\
\hline 1 & M & 24 & NSVT/SVT & 14 & $\mathrm{~F}$ & 46 & NSVT \\
\hline 2 & M & 23 & NSVT & 15 & $\mathrm{~F}$ & 30 & NSVT/SVT \\
\hline 3 & M & 22 & NSVT/SVT & 16 & M & 20 & NSVT/SVT \\
\hline 4 & M & 22 & NSVT & 17 & M & 44 & NSVT/SVT \\
\hline 5 & M & 62 & NSVT/SVT & 18 & $\mathrm{~F}$ & 38 & NSVT \\
\hline 6 & M & 36 & NSVT/SVT & 19 & $\mathrm{~F}$ & 42 & NSVT/SVT \\
\hline 7 & M & 43 & NSVT/SVT & 20 & M & 43 & NSVT/SVT \\
\hline 8 & M & 38 & NSVT/SVT & 21 & M & 26 & NSVT/SVT \\
\hline 9 & M & 52 & NSVT/SVT & 22 & M & 40 & NSVT/SVT \\
\hline 10 & M & 18 & NSVT/SVT & 23 & M & 13 & NSVT \\
\hline 11 & M & 20 & NSVT & 24 & M & 29 & NSVT/SVT \\
\hline 12 & M & 43 & NSVT/SVT & 25 & $\mathrm{~F}$ & 34 & NSVT/SVT \\
\hline 13 & $\mathrm{~F}$ & 31 & NSVT & 26 & $\mathrm{~F}$ & 38 & NSVT/SVT \\
\hline
\end{tabular}

dings were obtained from conventional electrocardiographs with a velocity of inscription of $25 \mathrm{~mm} / \mathrm{s}$ and standard calibration. Through a system of amplification of the recordings and definition of the points by a pair of compasses, the values (ms) of the QT intervals (from the beginning of the $\mathrm{Q}$ or $\mathrm{R}$ wave to the end of the $\mathrm{T}$ wave) were determined as the mean value of 3 successive complexes in the different electrocardiographic leads. For calculating the interval in the presence of an indefinite end of the T wave, we used the point of intersection of a tangent traced upon the descending branch of the wave with the baseline. The same process was used for excluding apparent $U$ waves. The degree of QTDp was defined through the difference between the longest and the shortest values of the interval observed among the diverse leads analyzed ${ }^{8}$. The patients' results were compared with those of a healthy control population similar in age and sex.

Antiarrhythmic drugs with a short half-life were suspended for a minimum period of 5 days prior to the examination. Patients on amiodarone underwent the study using the drug (table I). The protocol of the electrophysiological study included, in addition to the electrical mapping of the ventricular endocardium, the programmed electrical stimulation with the release of one, two, and three extrastimuli at the apex and right ventricular outflow tract, from the end of diastole until reaching the absolute refractory period of the cavity. Basic cycles of command of 500 and $430 \mathrm{~ms}$ and progressive shortening of the extrastimuli of $10 \mathrm{~ms}^{15}$ were also used. The endpoint of the test was triggering sustained ventricular tachycardia, defining patients with and without induction of sustained ventricular tachycardia. Data observed with QTDp were compared between the 2 populations.

In the clinical follow-up, deaths were computed through detailed information of the family, considering as sudden cardiac death, death occurring within the first hour from the beginning of the symptoms or of their aggravation, with or without the presence of witnesses ${ }^{16}$. The degree of QTDp was compared between the populations with and without sudden cardiac death.

All results were expressed as mean and 1SD. Differences between the results were assessed using variance analysis, considering $\mathrm{p}=0.05$ statistically significant. The ability of the method for defining patients at risk of sudden cardiac death was assessed through prognostic operational variables (sensitivity, specificity, positive and negative predictive values), using the ROC curve for optimizing its sensitivity and specificity. Prevalence of sudden death in the population, accuracy of the test, odds ratio, and the posttest probability of sudden death were also determined.

\section{Results}

The mean of the differences between the longest and the shortest QT intervals was $53.8 \pm 14.1 \mathrm{~ms}$ (ranging from 40 
to $80 \mathrm{~ms}$ ) for the patients and $35.0 \pm 10.6 \mathrm{~ms}$ (ranging from 20 to $60 \mathrm{~ms}$ ) for the healthy control group ( $\mathrm{p}=0.001)$. Table II shows these individualized results and figure 1 is a graphic representation of the mean differences in the degree of dispersion between the patients and the control group.

Twenty (77\%) patients underwent an electrophysiological study with confirmation of the diagnosis in all of them (presence of abnormal late potentials in diastole). Induction of monomorphic sustained ventricular tachycardia occurred through programmed ventricular stimulation in 8 patients (40\% - table II). The mean degree of QTDp for this group was $52.5 \pm 13.8 \mathrm{~ms}$ (ranging from 40 to $80 \mathrm{~ms}$ ) and for the group without induction it was $57.5 \pm 12.8 \mathrm{~ms}$ (ranging from 40 to $80 \mathrm{~ms}$ ), and no statistical significance was obtai$\operatorname{ned}(\mathrm{p}=0.420)$.

During a mean clinical follow-up of $41 \pm 29$ months (ranging from 10 to 120 months), 5 (22\%) sudden cardiac deaths occurred, 3 of them clearly related to physical activity, and all of them were witnessed. The mean time between the index electrocardiogram and the sudden death was 15.4 \pm 7.8 months (ranging from 10 to 28 months). The mean degree of dispersion for this group was $62.0 \pm 17.8 \mathrm{~ms}$ (ranging from 40 to $80 \mathrm{~ms}$ ), and for the group without sudden death it was $51.9 \pm 12.8 \mathrm{~ms}$ (ranging from 40 to $80 \mathrm{~ms}$ ); these results did not reach statistical significance $(\mathrm{p}=0.156)$. The type of medication used during clinical follow-up and the individualized results are shown in table II.

The best cutoff for optimization of sensitivity and specificity was $\geq 60 \mathrm{~ms}$. At this value, the method could identify patients prone to develop sudden cardiac death with the following percentages: sensitivity of $60 \%$, specificity of $52 \%$, positive predictive value of $23 \%$, and negative predictive value of $85 \%$. The prevalence of the event was $19.2 \%$. The accuracy of the test was $54 \%$. Odds ratio: positive of 1.7; negative of 0.6 .

\section{Discussion}

Experimental and clinical trials have shown a strict correlation between the increase in the degree of temporal dispersion of ventricular repolarization and the development of severe or fatal ventricular tachyarrhythmias. In the ' $60 \mathrm{~s}$, Han and Moe ${ }^{17}$ pointed out that the nonuniform recovery of the myocardial excitability represented an important factor triggering ventricular tachyarrhythmias, leaving the ventricles more vulnerable to ventricular fibrillation. These findings were later confirmed by other authors, such as Vassalo et al ${ }^{18}$, who analyzed the nonuniform recovery of left ventricular excitability, and Kuo et al ${ }^{19}$, who assessed the mechanisms involved in the genesis of ventricular arrhythmias related to dispersion of ventricular repolarization. Hii et al ${ }^{20}$ pointed out that the degree of dispersion of ventricular repolarization in precordial leads represents an important marker for the development of torsades de pointes in patients using drugs of the IA group, being also a relevant factor in acute myocardial infarction. During an acute coronary artery episode, patients developing ventricular fibrillation in the first 24 hours of evolution had a significant increase in the degree of QTDp as compared with

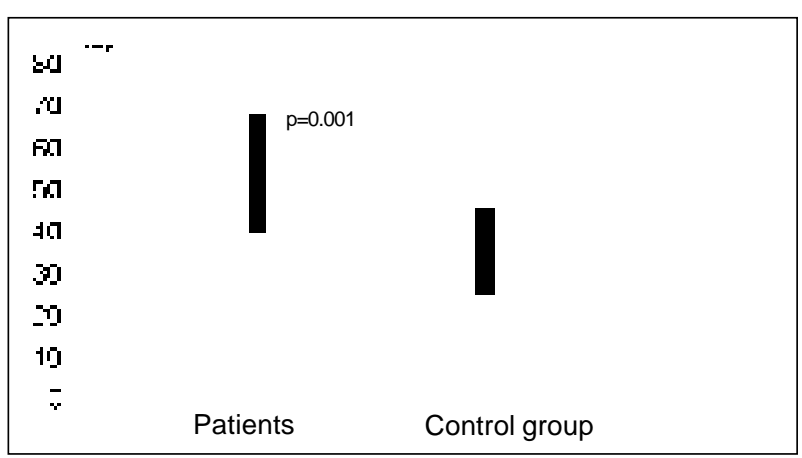

Fig. 1 - Graphic comparing the degrees of dispersion of ventricular repolarization observed in the patients and control group.

\begin{tabular}{|c|c|c|c|c|c|c|c|c|c|}
\hline \multicolumn{10}{|c|}{ Table II - Individualized results and clinical follow-up } \\
\hline Case & $\begin{array}{c}\text { Initial } \\
\text { QTDp }\end{array}$ & $\begin{array}{l}\mathrm{VT} \\
\text { Ind }\end{array}$ & $\begin{array}{l}\text { Clinical } \\
\text { follow-up }\end{array}$ & AA & Case & $\begin{array}{l}\text { Initial } \\
\text { QTDp }\end{array}$ & $\begin{array}{l}\text { VT } \\
\text { Ind }\end{array}$ & $\begin{array}{l}\text { Clinical } \\
\text { follow-up }\end{array}$ & AA \\
\hline 1 & 60 & No & SCD & St & 14 & 40 & No & $\mathrm{CT}$ & St \\
\hline 2 & 60 & No & SCD & $\mathrm{Bb}$ & 15 & 50 & No & $\mathrm{CT}$ & A \\
\hline 3 & 80 & No & $\mathrm{CT}$ & St & 16 & 50 & No & $\mathrm{SCD}$ & A \\
\hline 4 & 80 & No & SCD & St & 17 & 80 & - & $\mathrm{CT}$ & A \\
\hline 5 & 50 & No & $\mathrm{CT}$ & St & 18 & 70 & - & $\mathrm{CT}$ & A \\
\hline 6 & 40 & Yes & SCD & $\mathrm{Dm}$ & 19 & 50 & - & $\mathrm{CT}$ & A \\
\hline 7 & 40 & Yes & $\mathrm{CT}$ & $\mathrm{Mx}$ & 20 & 50 & Yes & $\mathrm{CT}$ & St \\
\hline 8 & 60 & Yes & $\mathrm{CT}$ & St & 21 & 80 & Yes & $\mathrm{CT}$ & St \\
\hline 9 & 60 & No & $\mathrm{CT}$ & St & 22 & 40 & - & $\mathrm{CT}$ & A \\
\hline 10 & 60 & No & $\mathrm{CT}$ & A & 23 & 60 & - & $\mathrm{CT}$ & A \\
\hline 11 & 50 & No & $\mathrm{CT}$ & St & 24 & 60 & Yes & $\mathrm{CT}$ & St \\
\hline 12 & 40 & No & $\mathrm{CT}$ & $\mathrm{St}$ & 25 & 50 & Yes & $\mathrm{CT}$ & A \\
\hline 13 & 60 & - & $\mathrm{CT}$ & $\mathrm{Bb}$ & 26 & 40 & Yes & $\mathrm{CT}$ & St \\
\hline
\end{tabular}

QTDp- dispersion of the QT interval in ms; VT Ind- induction of sustained ventricular tachycardia by programmed stimulation; AA- antiarrhythmic drug used in data collection; CT- clinical treatment; SCD- sudden cardiac death; A- amiodarone; Bb- beta-blocker; St- sotalol; Mx- mexiletine; Nm- no medication. 
those without this type of tachyarrhythmia ${ }^{21,22}$. Clinical applicability of the method was introduced by Day et al ${ }^{8}$, who showed that the difference between the longest and the shortest QT interval determined in the surface electrocardiogram was a marker of risk for the development of arrhythmias in patients with a prolonged QT interval. Later, Zabel et al ${ }^{23}$ observed an excellent correlation between the measures of the variables of dispersion obtained by external electrocardiogram and those measures obtained through interventricular monophasic potentials. Drugs such as beta-blockers will reduce the values of dispersion as shown in some specific situations ${ }^{24}$. Therefore, all studies indicate that the degree of temporal dispersion of ventricular repolarization represents an important marker for the development of ventricular tachyarrhythmias.

Few reports exist in the literature assessing QTDp as a predictive factor of induction of ventricular tachycardia in the electrophysiological study. Clay et al ${ }^{25}$ correlated QTDp of patients with induction of monomorphic and polymorphic ventricular tachycardia or ventricular fibrillation in the electrophysiological study and concluded that the method was not suitable for differentiating the type of induction (monomorphic or polymorphic). Sutter et al ${ }^{26}$ also observed no correlation between QTDp and induction of ventricular tachycardia by programmed ventricular stimulation. In our study, no difference in the degree of dispersion of ventricular repolarization occurred between the patients with and without induction. Therefore, current data suggest that independently from the type of disease, the method does not seem to be useful as a predictor of induction of sustained ventricular tachyarrhythmias by programmed ventricular electrical stimulation.

Most of the studies assessing the degree of QTDp as a marker of risk for sudden death involve patients with ischemic heart disease. The results of these studies have been controversial, especially the most recent ones. QTDp seems to have the capacity for differentiating patients who experienced ventricular fibrillation during an acute myocardial infarction ${ }^{21,22}$. In the Rotterdam Study ${ }^{27}$ involving 2,358 male patients and 3,454 female patients older than 53 years, $30 \%$ of whom were hypertensive and $13 \%$ of whom were postmyocardial infarction patients, QTDp proved to be an important predictive factor of overall mortality, of cardiac death, and of sudden death. In patients with depressed ventricular function, Fu et al ${ }^{28}$ observed that the measure of the dispersion of ventricular repolarization proved to be an important predictor of risk for sudden cardiac death. In patients with an implantable cardioverter-defibrillator, Grimm et al ${ }^{29}$ showed that QTDp was not a useful index for foretelling future arrhythmic events. Perkiomaki et al ${ }^{30}$ showed that the indices of variability of heart rate were superior to those of the QT interval for foretelling vulnerability to ventricular fibrillation. Zabel et al ${ }^{31}$, comparing patients with and without events, assessed 280 patients after myocardial infarction and showed that the method did not differentiate the 2 populations. Therefore, except for the Rotterdam Study with its significant sample of patients, most of the studies analyzing the predictive value of QTDp as a marker of arrhythmic events showed that the method has a low predictive power.

No studies on QTDp in arrhythmogenic cardiomyopathy of the right ventricle have been published. In our study, we were able to show that patients with arrhythmogenic right ventricular cardiomyopathy have a significant increase in the degree of QTDp as compared with a healthy control group. Using chest mapping with recordings of the surface potentials, Ambroggi et al ${ }^{32}$ managed to show an abnormal negative area in the anterior surface of the chest of patients with arrhythmogenic right ventricular cardiomyopathy, possibly related to the delay in the activation of the right ventricle. These authors also showed the existence of changes indicating nonuniform recovery of ventricular repolarization. This finding is said to correspond to the one we observed in the present study.

In regard to the ability of the method to predict arrhythmic events, our findings did not differ from those observed in most of the published reports involving distinct populations. The method did not differentiate patients with and without the terminal sudden event. It showed a very low predictive value (25\%) similar to the one observed with the isolated use of other methods, such as extrasystoles on Holter monitoring ${ }^{33}$ or signal-averaged electrocardiography ${ }^{34}$. With this method, patients with arrhythmogenic right ventricular cardiomyopathy showed an increase in the degree of QTDp as compared with healthy individuals. The significance of this finding, however, lacks prognostic value.

A large number of reasons exist to justify the low predictive value of QTDp for arrhythmic events. In addition to the limitations of the method, especially the correct definition of the end of the $T$ wave, the use of velocity recordings of $25 \mathrm{~mm} / \mathrm{s}$, in which small variations in its determination could introduce errors of up to $50 \mathrm{~ms}^{35}$ inside a short band of variation, the localized alterations of the activation and recovery forces through the myocardium cannot be totally detected on the surface electrocardiogram. This invalidates the method as a predictor of arrhythmic events. In the future with the introduction of more sophisticated techniques, such as spectral analysis of the $T$ wave for detecting alternation or chest mapping with recordings of surface potentials, the indices deriving from ventricular repolarization may better contribute as methods of prognostic assessment. 


\section{References}

1. Marcus F, Fontaine GH, Guirodon G, et al. Right ventricular dysplasia. A report of 24 adult cases. Circulation 1982; 65: 384-398.

2. Dalla Volta S. Arrhythmogenic cardiomyopathy of the right ventricle: thoughts on aetiology. Eur Heart J 1989; 10: 2-6.

3. Peters S, Weber B, Reil GH. Conventional electrocardiogram in arrhythmogenic right ventricular dysplasia cardiomyopathy and idiopathyc right ventricular outflow tract tachycardia. ANE 1996; 1: 400-09.

4. Thiene G, Nava A, Corrado D et al: Right ventricular cardiomyopathy and sudden death in young people. N Engl J Med 1988,318:1094-1104.

5. Corrado D, Thiene G, Nava A, et al. Sudden death in young competitive athletes: clinicopathologic correlation in 22 cases. Am J Med 1990; 898: 588-596.

6. Daliento L, Turrini P, Nava A, et al. Arrhythmogenic right ventricular dysplasia in young versus adult patients: similarities and differences. J Am Coll Cardiol 1995; 25: 655-64

7. Corrado D, Basso C, Thiene G. Sudden death. In: Nava A, Rossi L, Thiene G. Eds. Arrhythmogenic Right Ventricular Cardiomiopathy/Dysplasia. Lausanne: Elsevier, 1997: 36-45.

8. Day CP, McComb JM,Campbell RWF. QT dispersion: an indication of arrhythmia risk in patients with long QT intervals. Br Heart J 1990; 63: 342-4.

9. Zareba W, Moss AJ, Le Cessie S. Dispersion of ventricular repolarization and arrhythmic cardiac death in coronary artery disease. Am J Cardiol 1994; 74: 550-3.

10. Moreno FL, Villanueva T, Karagounis LA, Anderson J. Reduction in QT interval dispersion by successful thrombolytic therapy in acute myocardial infarction. Circulation 1994; 90: 94-100.

11. Kinoshita O,Fontaine G, Rosas F, et al. Time and frequency domain analysis of the signal-averaged ECG in patients with arrhythmogenic right ventricular dysplasia. Circulation 1995; 91: 715-27

12. Mehta D, Goldman M, David O, Gomes JA. Value of quantitative measurement of signal-averaged electrocardiographic variables in arrhythmogenic right ventricular dysplasia: correlation with echocardiographic right ventricular cavity dimensions. J Am Coll Cardiol 1996; 28: 713-19.

13. McKenna WJ, Thiene G, Nava A, et al: Diagnosis of arrhythmogenic right ventricular dysplasia/cardiomyopathy. Task force of the working group of myocardial and pericardial disease of the European Society of Cardiology and of scientific council on cardiomyopathies of the International Society and Federation of Cardiology. Br Heart J 1994; 71: 215-18.

14. Wichter $T$, Lentschig MG, Reimer $P$, et al. Magnetic resonance imaging. In: Nava A, Rossi L, Thiene G. Arrhythmogenic Right Ventricular Cardiomyopathy/Dysplasia. Lausanne: Elsevier, 1997: 269-84.

15. Brugada P, Wellens HJJ. Programed electrical stimulation of the human heart: general principles. In: Josephson ME, Wellens HJJ, Eds. Tachycardias: Mechanisms, Diagnosis and Treatment. Philadelphia: Lea Febiger, 1984: 61.

16. Epstein AE, Carlson MD, Fogoros RN, et al: Classification of death in arrhythmogenic trials. J Am Coll Cardiol 1996; 27: 433-42.

17. Han J, Moe GK. Nonuniform recovery of excitability in ventricular muscle. Circ Res 1964; 14: 44-60.

18. Vassalo JA, Cassidy DM, Kindwall KE, et al. Nonuniform recovery of excitability in the left ventricle. Circulation 1988; 78: 1365-72.

19. Kuo CS, Munakata K, Reddy CP, Surawicz B. Characteristics and possible mecha- nism of ventricular arrhythmia dependent on the dispersion of action potential durations. Circulation 1983; 67: 1356-67.

20. Hii JTY, Wyse GD, Gillis AM, et al. Precordial QT interval dispersion as a marker of torsade de pointes. Disparate effects of class IA antiarrhythmic drugs and amiodarone. Circulation 1992; 86: 1376-82.

21. Loo A, Arendts W, Hohnloser SH. Variability of QT dispersion measurements in the surface electrocardiogram in patients with acute myocardial infarction and normal subjects Am J Cardiol 1994; 74: 1113-18.

22. Rashba EJ, Zareba W, Moss AJ. The relation of QT dispersion to spontaneous ventricular arrhythmias during the acute phase of myocardial infarction ANE 1998; 3: 119-24.

23. Zabel M, Lichtlen PR, Haverich A, Franz MR. Comparison of ECG variables of dispersion of ventricular repolarization with direct myocardial repolarization measurements in the human heart. J Cardiovasc Electrophysiol 1998; 9: 127984.

24. Priori SG, Napolitano C, Diehl L, Schwartz PJ. Dispersion of the QT interval: a marker of therapeutic efficacy in the idiopathic long QT syndrome. Circulation 1994; 89: 1681-9.

25. Clay A, Dreifus LS, Zaim S, Kutalek SP. Can dispersion of the QT interval identify inducible monomorphic versus polymorphic ventricular tachycardia? ANE 1998; 3: 20-4.

26. Sutter J de, Tavernier R, Wiele CV, et al. QT dispersion is not related to infarct size or inducibility in patients with coronary artery disease and life threatening ventricular arrhythmias. Heart 1999; 81: 533-8.

27. Bruyne MC, Hoes AW, Kors JA, et al. QTe dispersion predicts cardiac mortality in the elderly. The Rotterdam study. Circulation 1998; 97: 467-72.

28. Fu GS, Meissner A, Simon R. Repolarization dispersion and sudden cardiac death in patients with impaired left ventricular function. Eur Heart J 1997; 18 281-9.

29. Grimm W, Steder U, Menz V, Maisch B. Predictive value of QT dispersion for ventricular tachyarrhythmias in patients with implantable cardioverter defibrillator. ANE 1996; 1: 419-22.

30. Perkiomaki JS, Huikuri HV, Koistinen JM, et al. Heart rate variability and dispersion of QT interval in patients with vulnerability to ventricular tachycardia and fibrillation after previous myocardial infarction. J Am Coll Cardiol 1997; 30: 1331-8.

31. Zabel M, Klingenheben T, Franz MR, Hohnloser SH. Assessment of QT dispersion for prediction of mortality or arrhythmic events after myocardial infarction. Circulation 1988; 97: 2543-50.

32. Ambroggi L, Aimè E, Ceriotti $\mathrm{C}$, et al. Mapping of ventricular repolarization potentials in patients with arrhythmogenic right ventricular dysplasia: principal component analysis of the ST-T waves. Circulation 1997; 96: 4314-18.

33. Hartikainen JEK, Malik M, Staunton A, et al. Distinction between arrhythmic and nonarrhythmic death after acute myocardial infarction based on heart rate variability, signal-averaged electrocardiogram, ventricular arrhythmias and left ventricular ejection fraction. J Am Coll Cardiol 1996; 28: 296-304.

34. Hohnloser SH, Franck P, Klingenheben T, et al. Open infarct artery, late potentials, and other prognostic factors in patients after acute myocardial infarction in the thrombolytic era. Circulation 1994; 90: 1747-56.

35. Coumel P, Maison-Blanche P, Badilini F. Dispersion of ventricular repolarization: Reality? Illusion? Significance? Circulation 1998; 97: 2491-3. 